\title{
Parlamento e poteri legislativi del Governo in Italia: l'abuso del decreto d'urgenza (decreto-legge)
}

Alfonso Celotto

I. Premessa: il decreto-legge nella Costituzione italiana.

Il decreto d'urgenza (decreto-legge), per quanto non previsto, anzi implicitamente vietato dallo Statuto Albertino (1848), finì con l'affermarsi nella prassi del Regno d'Italia, con abusi anche notevoli. Tali abusi non furono contenuti dalla l. 31 gennaio 1926, n. 100, in cui all'Art. $3^{\circ}$ si riconosceva al Governo la facoltà di emanare norme aventi forza di legge "nei casi straordinari, nei quali ragioni di urgente ed assoluta necessità lo richiedano", assoggettando il controllo di tali presupposti solo al Parlamento e disponendo che in caso di mancata conversione entro due anni dalla pubblicazione il decreto cessasse di aver vigore ex nunc.

Anzi negli anni del regime fascista, anche in ragione del particolare rafforzamento dell'Esecutivo, la decretazione d'urgenza continuò ad incrementarsi fino a divenire la forma principale di produzione normativa.

Conscia delle precedenti degenerazioni, l'Assemblea costituente repubblicana, dopo un serrato dibattito, scelse di disciplinare puntualmente il decreto-legge, circondandolo di freni e limitazioni, al pari della delegazione legislativa (Art. $76^{\circ}$ Cost.), in un sistema delle fonti incentrato sulla legge parlamentare (Art. $70^{\circ}$ Cost.).

Così l'Art. 770 Cost. ammette che "quando, in casi straordinari di necessità e d'urgenza, il Governo adotta, sotto la sua responsabilità, provvedimenti provvisori con forza di legge deve il giorno stesso presentarli per la conversione alle Camere che, anche se sciolte, sono appositamente convocate e si riuniscono entro cinque giorni». 
Oltre alla responsabilità governativa ed alla immediata convocazione delle Camere, il Costituente pensò di contenere l'istituto anche mediante il conferimento di un'efficacia precaria a tali decreti che "perdono efficacia sin dall'inizio, se non sono convertiti in legge entro sessanta giorni dalla loro pubblicazione" (come dispone il successivo terzo comma dell'Art. $7^{\circ}$ Cost.).

$\mathrm{Si}$ tratta quindi di una fonte provvisoriamente dotata di forza e valore di legge, che il Governo - e più precisamente il Consiglio dei Ministri - può adottare solo alla ricorrenza dei presupposti delineati dalla "clausola generale" contenuta nella Carta fondamentale (casi straordinari di necessità ed urgenza).

Se il decreto non viene convertito in legge entro sessanta giorni perde efficacia fin dall'inizio e solo le Camere possono regolare con legge i rapporti giuridici sorti medio tempore. Fin dall'inizio - malgrado diverse opinioni contrarie - la prassi parlamentare ha ammesso la possibilità di emendamenti, cioè di modificazioni al testo del decreto in sede di conversione, che entrano in vigore - ai sensi del $5^{\circ}$ comma dell'Art. $15^{\circ} \mathrm{l} .400 / 88$, che ha posto fine a diverse incertezze - il giorno successivo alla pubblicazione della legge di conversione, salvo quest'ultima disponga diversamente.

Nei primi anni di vita dell'ordinamento repubblicano, lo strumento del decreto-legge è stato usato in maniera moderata.

E' a partire dalla fine degli anni ' 60 che - grazie anche alle condiscendenti elaborazioni dei costituzionalisti - la decretazione d'urgenza comincia progressivamente ad incrementarsi, mediante una interpretazione sempre più estensiva dei presupposti costituzionali. Mediante distorsioni quali la reiterazione - consistente nella riemanazione del decreto, in caso di mancata conversione o, a volte, anche di riffuto di conversione - l'adozione di decreti omnibus, la facilità di inserire emendamenti in sede di conversione, il fenomeno ha assunto dimensioni sempre più massicce'.

1 Per una più ampia analisi storica e sistematica dell'istituto, ci sia consentito rinviare a Celotto, L' «abuso» del decreto-legge, Padova, 1997, p. 101 y ss. 


\section{Uso e abuso del decreto-legge dalla X alla XIII legislatura repubblicana}

\subsection{Un abuso parossistico.}

Negli ultimi decenni si è potuto assistere ad un grave fenomeno di crisi della legge, che ha perduto quel ruolo di guida del sistema che tradizionalmente le spettava quale fonte primaria per eccellenza ${ }^{2}$. Nella produzione è così, la legge parlamentare è stata affiancata e sopravanzata dalle fonti legislative governative, tanto che il decretolegge si è "trasformato in uno strumento ordinario di legiferazione, con qualche tendenza a divenire lo strumento prevalerte ${ }^{3}$.

Alla fine degli anni ' 80 , il sempre maggiore indebolimento delle maggioranze governa-tive ${ }^{4}$, il cattivo funzionamento del Parlamento ${ }^{5}$, la crescente instabilità politica hanno via via reso sempre più neces-sario il ricorso ad uno strumento di colegislazione, di codeterminazione politica, di negoziato tra governo, maggioranza e opposizione quale nella prassi è divenuto il decreto-legge.

Conseguentemente, il ricorso alla decretazione legislativa del governo - solo nominalisticamente d'urgenza - si è incrementato notevolmente, rendendo questa forma di produzione «un modo ordinario e parallelo, se non prevalente, di legiferazione a fianco o addirittura in sostituzione di quello parlamentare» ${ }^{6}$.Il record di 302 decreti-legge raggiunto nella IX legislatura (1983-1987) viene ampiamente superato nella X legislatura (1987-1992), in cui si emanano 466 decreti. Ma il dato che esplode veramente è quello della mancata conversione in legge. Nelle prime sette legislature la percentuale di decreti-legge convertiti si era sempre mantenuta al di sopra dell' $80 \%$, calando al $61 \%$ nella

2 In proposito cfr., di recente, Modugno - Celotto - Ruotolo, Considerazioni sulla crisi della legge, in Studi Parlamentari e di Politica Costituzionale, 1999, pp. 125126, y p. 7 y ss.

3 Così Ciaurro, "Decreto-legge», in Enc. Giuridica Treccani, X, Roma, 1988, $\$$ 2.2.3.

4 Zagrebelsky, Manuale di diritto costituzionale, I) Il sistema delle fonti del diritto, Torino, 1990, p. 178.

5 Manzella, Il Parlamento, nuova ediz., Bologna, 1991, p. 274.

6 Modugno, - Nocilla, "Crisi della legge e sistema delle fonti”, in Dir. e Società, 1989, p. 425. 
VIII legislatura (1979-1983). E' nelle successive due legislature che questo dato crolla, arrivando prima al $45 \%$ e poi al $40 \%$, con un corrispondente incremento notevole delle reiterazioni, che cominciano a divenire pratica abituale in esito alla mancata conversione.

Tali campanelli di allarme rendono evidente l'esigenza di interventi contenitivi, che puntualmente giungono sia in sede normativa, sia in sede giurisdizionale. Sul primo versante, viene emanato l'Art. $15^{\circ}$ della legge $n^{\circ} 400$ del 1988, enucleando una articolata serie di limiti contenutistici e funzionali al decreto-legge e si modifica l'Art. $78^{\circ} \mathrm{Reg}$. Senato, ampliando il parametro della verifica preliminare di legittimità, da parte della Commissione Affari costituzionali del Senato, anche ai «limiti stabiliti dalla legislazione vigente» (e, quindi, proprio al disposto della 1. n. 400 del 1988). Sul secondo versante la Corte costituzionale, con la sent. $\mathrm{n}^{\circ} 302$ del 1988 , condanna con durezza la prassi della reiterazione, dichiarando incostituzionale un decreto-legge più volte riproposto per lo "svuotamento sostanziale» delle competenze regionali che ne era conseguito e lanciando un secco monito sulla illegittimità di tale prassi.

Di fatto, però, questi «rimedi» - al pari degli impegni ripetutamente assunti dai Presidenti del Consiglio in occasione delle proprie dichiarazioni programmatiche alle Camere - producono scarsi effetti pratici. L'Art. $15^{\circ}$ della legge n. 400 del 1988 - che pure contiene prescrizioni senz'altro idonee ad un efficace contenimento dell'istituto - non viene ritenuto vincolante rispetto ai successivi decreti-legge, anche in forza delle quasi unanimi perplessità dottrinarie circa l'equiparazione gerarchica tra fonti ${ }^{7}$. La Corte costituzionale, invece, non dà seguito alle affermazioni della sent. $\mathrm{n}^{\circ} 302$ del 1988 - che pure qualche «ripercussione» avevano avuto - proseguendo in una giurisprudenza oscillante, quasi "timorosa»".

E' così che, con il passaggio agli anni ' 90 , favorita dalla ancor maggiore instabilità politica e dalla scarsa efficienza tanto dei controlli

7 Per tutti, Pace, "I ridotti limiti della potestà normativa del Governo nella legge $n^{\circ} 400$ del 1988", in Giur. Cost., 1988, II, p. 1483 y ss.; Carlassare, "Prime impressioni sulla nuova disciplina del potere regolamentare prevista dalla legge $n^{\circ} 400$ del 1988 a confronto con principio di legalità", in Giur. Cost., 1988, II p. 1482; in senso contrario, isolatamente, Modugno, voce "Validità (diritto costituzionale)», in Enc. Dir., XIVI, Milano, 1993, p. 61 y ss.

8 Cfr. ord. n. 225 del 1992; e sentt, n. 364 del 1993; e nn. 40 e 263 del 1994. 
politici che di quelli giurisdizionali ${ }^{9}$, la decretazione legislativa d'urgenza - pur a fronte di compagini governative di differente composizione, quasi a voler confermare la sostanziale inevitabilità del fenomeno ${ }^{10}$ continua a crescere in maniera incessante, riuscendo a raggiungere livelli che sembravano impensabili anche solo qualche anno prima (si pensi, ad es., che nel solo 1994 si sono emanati 327 decreti legge, più che in tutto il quadriennio della IX legislatura).

Nella XI (1992-1994) e nella XII (1994-1996) legislatura - peraltro di durata molto breve - si incrementano ancora, in maniera esponenziale, non solo il numero dei decreti-legge - che raggiungono quasi la media di uno al giorno (si pensi ai 296 decreti-legge del 1995 e ai 361 del 1996, a fronte dei 3 del 1949 e 2 del 1950) - ma anche tutti gli altri indicatori patologici (in particolare nelle due menzionate legislature, le percentuali di conversione crollano al $24.2 \%$ e al $16.9 \%$, mentre quelle di reiterazione raggiungono il $66.9 \%$ e il $76.0 \%$ ).

In tal modo il decreto-legge - da fonte eccezionale o, quanto meno, alternativa e parallela nella produzione normativa - è diventato addirittura la fonte principale, quasi esclusiva di produzione del diritto, tanto da arrivare a sopravanzare quella parlamentare (basti rilevare, ad es., che nel 1996 sono stati emanati 533 atti aventi forza di legge, a loro volta ripartiti in 133 leggi, 39 decreti legislativi e 361 decreti-legge).

La patologia del decreto-legge non emergeva solamente in termini quantitativi ma anche, inevitabilmente - oltre un certo limite la "quantità» ridonda comunque sulla "qualità»" - anche sul piano qualitativo. Infatti, puntualmente, la produzione decretizia degli ultimi anni ha mostrato in maniera evidente $i$ segni di una «incontenibile rilassatezza di costumi ${ }^{12}$, non solo incidendo in ambiti ad essi ritenuti naturalmente preclusi, ma anche intervenendo in maniera sempre più scorretta, disordinata, tortuosa, farraginosa ${ }^{13}$.

9 Su tali cause Sorrentino - Caporali, voce "Legge (Atti con forza di)", in Dig. disc. pubbl., IX, Torino, 1994, p. 123; Pace, "Divagazioni suidecreti-legge non convertiti», in Pol. del diritto, 1995, r. 396, D'Auria, "La "funzione legislativa" dell'amministrazione", in Riv. Trim. Dir. Pubbl., 1995, p. 724 y s.

10 Cfr. Silvestri, "Alcuni profili problematici dell'attuale dibattito sui decreti-legge», in Pol. del diritto, 1996, p. 422.

11 Cfr., con riferimento più generale alla produzione legislativa, già Carnelutti, "La crisi della leggew, in Riv. Dir. Pubbl., 1930, I, p. 429.

12 Silvestri, Alcuni profili problematici dell'attuale dibattito sui decreti-legge, cit., P. 421. 


\subsection{Il «male oscuro» della reiterazione.}

Il punto di massima emersione dell'abuso del decreto-legge va comunque rinvenuto nella reiterazione, non a caso definita uno "scandalo» ${ }^{14}$, una "pratica cancerosa» che froda la Costituzione ${ }^{15}$, e puntualmente indicata quale «il fenomeno centrale che ha permesso la radicale modificazione della funzione del decreto-legge nel sistema delle fontis ${ }^{16}$.

Dalla metà degli anni ' 80 , a fronte dell'aumento del numero dei decreti e quindi della loro mancata conversione, è cominciata a divenire abituale e sistematica la pratica - di là da fenomeni più scorretti come la reiterazione di decreti-legge respinti dalle Camere - di ripresentare, alla vana scadenza del termine costituzionale per la conversione, un nuovo decreto riproduttivo dei contenuti del precedente: si pensi che nella VII legislatura su 15 decreti-legge decaduti ne sono stati reiterati 9 (pari al 60\%), nella VIII legislatura si è passati alla reiterazione di 71 decreti su 93 decaduti (il 76,3\%), nella IX di 134 su 139 (96,4\%), nella X di 224 su 264 (84,8\%), nella XI di 328 su $363(90,3 \%)$ e nella XII di 546 su $558(97,8 \%)$.

L'enorme quantità di decreti reiterati ha creato notevolissimi problemi pratici, soprattutto per l'evoluzione della prassi in senso costituzionalmente più corretto ma con effetti pratici paradossali. Fino al 1988 il successivo decreto-legge della "catena" era solito contenere - illegittimamente - la «sanatoria» degli effetti dei precedenti decreti non convertiti; dopo di allora, invece, anche in ossequio alla prescrizione

13 Si pensi ad es., all' "affiancamento" di decreti-legge e disegni di legge ordinari con il medesimo contenuto, a decreti che modificano leggi di conversione a pochi giorni dalla loro approvazione al fine di "imporre» al Parlamento una determinata disciplina, all'emanazione nello stesso decreto di due disposizioni di modifica testuale, in maniera diversa, di una disposizione precedente: per una analisi di queste fattispecie ci sia consentito rinviare a Celotto, L' "abuso" del decreto-legge, cit., p. 294 y ss.

14 Silvestri, "Sulla conversione in legge dei decreti-legge iterati e reiterati», in $\mathrm{Aa} . \mathrm{Vv}$., I decreti-legge non convertiti, Milano, 1996, p. 161.

15 Rescigno, "Le tecniche legislative oggi in Italia", in Visintini, (a cura di), Analisidi leggi campione. Problemi di tecnica legislativa, Padova, 1995, P. 739.

16 Così Modugno, "Analisi delle disposizioni della legge 400188 relative alla potestà legislativa del governo. Considerazioni critiche", in Scritti in onore di G. Guarino, Milano, 1998 , III, p. 64; v. anche Sorrentino, "Il decreto-legge non convertito", in Pol. del diritto, 1995, P. 422. 
dell'Art. 150, lett. d), 1. 400/88, è divenuto abituale collocare nel secondo comma dell'Art. $1^{\circ}$ del disegno di legge di conversione, quale clausola di stile $e^{17}$, la convalida degli effetti dei precedenti decreti. La prassi precedente, pur ponendosi in palese contrasto con l'Art. $77^{\circ}$, ult. co., Cost., consentiva una sostanziale continuità degli effetti del decreto-legge vigente con quelli dei decreti precedentemente non convertiti; invece, questo apparente ritorno alla lettera della Costituzione ha dato vita a rilevantissime difficoltà dal punto di vista pratico: la «nuova» forma di sanatoria, infatti, non è contenuta in un atto avente forza di legge, per cui - in attesa della conversione in legge dell'ultimo decreto della "catena", che spesso si fa attendere per molti mesi (essendosi arrivati a reiterare decreti anche per 29 volte!) - si viene a creare il paradosso ${ }^{18}$ : gli effetti prodotti dai decreti precedenti decadono e così si produce un buco nell'efficacia delle disposizioni, tanto più ampio e pernicioso quanto più è insistita la reiterazione!

Tale prassi, conseguentemente, lede in maniera gravissima il principio della certezza del diritto ${ }^{\prime 9}$, in quanto - allungando di fatto la provvisorietà - comporta che la maggior parte dell'attività giuridica resti disciplinata precariamente, e spesso in maniera disomogenea, dati gli aggiustamenti contenuti nel singolo decreto ad ogni reiterazione, sovente al fine di recepire gli emendamenti già approvati in sede di iter parlamentare di conversione, per mesi e mesi.

In tal modo il decreto-legge viene ad essere profondamente e radicalmente snaturato, in quanto la reiterazione sistematica ed insistita che dà vita ad un vero e proprio procedimento alternativo di produzione normativa - gli fa perdere la sua intrinseca e naturale provvisorietà ${ }^{20}$. Del resto, il decreto-legge ripetutamente reiterato crea effetti sempre più irreversibili ${ }^{21}$, importando nell'opinione pubblica un affidamento sulla

17 Come esattamente definita da Paladin, Le fonti del diritto italiano, Bologna, 1996, p. 251.

18 Cfr. F. Crisafulli, "Appendice di aggionnamento" a V. Crisafulli, Lezioni di diritto costituzionale, VI ediz., Padova, 1993, p. 17.

19 Cfr. lucidamente Cicconetti, "Nuovi elementi in tema di reiterazione di decreti-legge", in Giur. Cost., 1989, II, p. 1492.

$20 \mathrm{Cfr}$. Ruggeri, Fonti e norme nell'ordinamento e nell'esperienza costituzionale, Torino, 1993, p. 335.

21 Cfr. Ainis, "Gli effetti irreversibili del decreto-legge", in Id., Le parole e il tempo della legge, Torino, 1996. 
sua stabilità ${ }^{22}$ - come emerge nitidamente da alcune pronunce giurisprudenziali ${ }^{23}$ - e, soprattutto, coartando il Parlamento non tanto alla conversione in legge che resta pur sempre libera ma, prima, a pronunciarsi sull'atto, per approvarlo o modificarlo o respingerlo, non potendolo invece di fatto «ignorare» all'infinito ${ }^{24}$, e, poi, specificamente, alla sanatoria degli effetti comunque prodotti dai decreti non convertiti ${ }^{25}$. Solo l'intervento parlamentare può dare saldezza al "castello di carte» che si è venuto a formare mediante la «catena» dei decretilegge, la cui decadenza ex tunc produrrebbe effetti disastrosi.

In tal modo, addirittura, si capovolge la prima parte del terzo comma dell'Art. $77^{\circ}$ Cost., in quanto - in buona sostanza - i decreti-legge non possono più perdere "efficacia fin dall'inizio, se non sono convertiti in legge entro sessanta giorni dalla loro pubblicazione ${ }^{26}$.

\subsection{L'inevitabile intervento contenitivo della Corte} costituzionale: la sent. n. 360 del 1996 e la prassi successiva.

A livello di sistema, la decretazione legislativa d'urgenza aveva ormai assunto un ruolo troppo ampio, per cui nel corso del $1996 \mathrm{i}$ tentativi di contenimento si sono intensificati, anche sotto la spinta di una sorta di ribellione organizzata dei giudici ordinari - palese sintomo dell'intollerabilità della situazione - $i$ quali, soprattutto in materia penale, hanno cominciato a non applicare più i decreti-legge sistematicamente reiterati, sollevando anzi altrettanto sistematicamente questione di legittimità costituzionale per violazione dell'Art. $77^{\circ}$ Cost. ${ }^{27}$.

22 Cfr., per tutti, Paladin, "Atti legislativi del Governo e rapporti tra i poteri", in Quad. Cost., 1996, p. 26.

23 Per quanto teoricamente assurdo, la giurisprudenza è arrivata ad ammettere l'efficacia sanante della reiterazione in sé; cfr., ad es., Cass. Pen., sez. V, 22 aprile 1993, Villa, in Cass. Pen., 1995, p. 1518.

24 Cfr. Berti, Manuale di interpretazione costituzionale, III ediz., Padova, 1994, p. 175.

25 Cfr. già Tarchi, "Incompetenza legislativa del Governo, interposizione del Parlamento e sindacato della Corte costituzionale», in Giur. Cost., 1988, II, p. 947.

26 Sul punto Sorrentino, Il decreto-legge non convertito, cit., p. 422.

27 Per una più ampia analisi di questo rudimentale, ma efficace rimedio - attivato soprattutto riguardo alla catena di decreti-legge sullo smaltimento dei rifiuti, fatti oggetto di oltre centocinquanta questioni di legittimità costituzionale - ci sia consentito rinviare Celotto, "Sarà il giudice ordinario a limitare la reiterazione dei decreti-legge?», in Dir. e Società, 1995 , p. 555 y ss. 
Dapprima, il governo Prodi fin dal suo insediamento - su impulso di un forte monito del Presidente della Repubblica ${ }^{28}$ e dei segnali di maggior rigore da parte della Corte costituzionale - ha cercato di ridimensionare la massa di 94 decreti-legge pendenti ricevuti in "eredità» dalla precedente legislatura. I tentativi a cio finalizzati - mediante l'accorpamento di alcuni decreti, la decadenza di altri, accompagnata da specifici disegni di legge di sanatoria, e l'autolimitazione nell'emanazione di nuovi decreti, ai soli casi strettamente necessari avevano prodotto risultati solo parziali, anche se comunque si è riusciti ad arrivare all'inizio del mese di settembre 1996 a soli 53 decreti-legge pendenti in attesa di conversione.

Su altro versante, in sede di dibattito sulle riforme istituzionali, è stabilmente emersa l'esigenza - peraltro mai portata a compimento - di riformare l'Art. $77^{\circ}$ Cost., soprattutto indicando le materie ammesse alla decretazione governativa d'urgenza (sicurezza nazionale, calamità naturali, provvedimenti finanziari) e aggiungendo una serie di preclusioni funzionali (generalmente delega legislativa, reiterazione di decreti-legge e sanatoria dei loro effetti, ripristino di norme annullate per vizi non attinenti al procedimento ${ }^{29}$.

Ad ogni modo, un reale effetto di contenimento degli abusi del decretolegge si è verificato solo a seguito di quella che - potremmo ritenere la più importante decisione mai emanata dalla Corte costituzionale in tema di decreti-legge.

Con la sent. n. 360 del 1996, la Corte ha finalmente portato a conseguenze rigorose i mutamenti di giurisprudenza di cui aveva dato ripetuti segnali nei mesi precedenti, arrivando a dichiarare l'illegittimità costituzionale della reiterazione sulla base dell'assunto che il «decretolegge iterato o reiterato - per il fatto di riprodurre (nel suo complesso o in singole disposizioni), il contenuto di un decreto-legge non convertito, senza introdurre variazioni sostanziali - lede la previsione costituzionale sotto più profili, ${ }^{30}$.

28 Cfr. il messaggio inviato il 30 maggio 1996 dal pres. Scalfaro al presidente del Consiglio incaricato subito prima della votazione sulla fiducia al governo, pubblicato in testo integrale ne Il Sole 24 ore del 5 giugno 1996.

29 Per un'analisi delle proposte di riforma cfr. Romboli, "Le vicende della decretazione d'urgenza negli anni 1995-1997 tra Corte costituzionale e ipotesi di revisione dell'Art. $7^{\circ}$ Cost.". in Studi in onore di L. Elia, Milano, 1999, II.

$30 \$ 4$ Considerato in diritto. 
A ben vedere si tratta di una condanna non incondizionata né totale, in quanto la Corte, da un lato - guardando al passato - gradua gli effetti temporali della propria pronuncia, facendo espressamente salvi - non senza dar adito a perplessità - «gli effetti dei decreti-legge iterati o reiterati già convertiti in legge» e di quelli «la cui conversione risulti attualmente in corso, ove la stessa intervenga nel termine fissato dalla Costituzione»; e dall'altro - proiettandosi verso il futuro e recependo gli spunti di parte della dottrina $\mathrm{a}^{31}$ - ammette che il Governo, in caso di mancata conversione di un decreto-legge, possa riprodurre, con un nuovo decreto, il contenuto normativo dell'intero testo o di singole disposizioni del decreto non convertito, ove il nuovo decreto «risulti fondato su autonomi (e, pur sempre, straordinari) motivi di necessità ed urgenza, motivi che, in ogni caso, non potranno essere ricondotti al solo fatto del ritardo conseguente alla mancata conversione del precedente decreto" oppure ove sia "caratterizzato da contenuti normativi sostanzialmente diversi ${ }^{32}$.

Ad ogni modo, questa presa di posizione del giudice costituzionale è riuscita a frenare quasi miracolosamente gli abusi del decreto-legge.

Dalla fine del 1996 è infatti scomparsa la prassi della reiterazione. Il numero dei decreti-legge ha quindi subito una drastica contrazione (si pensi se nei primi 4 mesi del 1996 il Governo aveva emanato 156 decreti-legge, di cui ben 118 reiterazioni di precedenti decreti non convertiti; nel corrispondente periodo del 1997, sono stati emanati invece solo 17 decreti-legge, tutti «nuovi»; e nel primo quadrimestre del 1998, soltanto 8 decreti, anch'essi «nuovi»), anche se il ricorso alla decretazione d'urgenza continua a scontare comunque una scarsa rispondenza ai presupposti costituzionali ${ }^{33}$, nel senso che si continuano ad emanare decreti-legge per far fronte a situazioni di cui si può fondatamente opinare la reale straordinaria necessità ed urgenza ${ }^{34}$.

31 Cfr., per tutti, già Paladin, L., "Art. $77^{\circ}$ ", in Commentario alla Costituzione a cura di G. Branca, Bologna-Roma, 1977, p. 62 y s.

$32 \$ 5$ Considerato in diritto.

33 Non risolta neppure dalle più recenti modifiche al regolamento della Camera: cfr. nuovo testo Art. 96\%-bis Reg. Camera, modificato nel 1997, su cui Nasi, L'Art. 960-bis del regolamento della Camera ed il procedimento di conversione dei decreti-legge, in Rass. Parl., 2001, p. 456 yss.

34 Sulla prassi della decretazione d'urgenza dopo la sent. n. 360 del 1996 cfr. Simoncini, "Il sistema delle fonti tra governo e parlamento dopo la sentenza n. 360/96 della Corte costituzionale», in Rass. Parl., 1997, p. 989 y ss.; e Maccabiani, "Le reiterazioni dei decreti-legge successive alla sentenza 360196 della Corte costituzionale», in Rass. Parl, 2001, p. 423 y ss. 


\subsection{L'abuso del decreto-legge nella forma di governo: un sintomo inequivocabile della crisi del sistema delle fonti.}

L'abuso del decreto-legge - e, più in generale l'ampliamento dei potersi normativi dell'esecutivo - integra un "fenomeno che tende ad espandersi quando l'ordinamento versa in situazioni di crisi; [e] a ridursi quando la vita dell'ordinamento torna a scorrere nell'alveo della normalità ${ }^{35}$, atteggiandosi ad epifenomeno di un problema molto più complesso, che ridonda sull'assetto del sistema delle fonti del diritto e, quindi, sulla configurazione della stessa forma di governo.

Del resto, non è pensabile che la sostanziale appropriazione del potere normativo da parte del Governo costituisca un fenomeno isolato, in quanto il tipo di equilibrio operante - in un determinato paese e in un dato momento storico - tra poteri dell'esecutivo e poteri del legislativo costituisce uno dei criteri, se non il principale, per determinare la forma di governo in esso operante ${ }^{36}$.

A livello di sistema l'ampliamento ed il consolidarsi di poteri normativi primari dell'esecutivo - verificatosi in Italia come all'estero in diversi momenti storici - porta ad un sostanziale capovolgimento di ruoli tra Parlamento e Governo ${ }^{37}$ : la competenza normativa non è più ripartita secondo il criterio-base dell'enunciazione delle regole di principio da parte dell'uno e della loro esecuzione da parte dell'altro, in quanto entrambi arrivano a disporre del potere di emanare le norme ritenute più opportune, utilizzando due procedimenti che divergono soltanto per la maggiore o minore celerità, tanto da rendere ordinaria la fonte straordinaria (decreto-legge) e straordinaria quella ordinaria (legge parlamentare). Nel far ciò la prassi non solo ha eluso i limiti posti dall'Art. $77^{\circ}$ Cost., ma addirittura - paradossalmente - è arrivata a creare un nuovo tipo di decreto-legge, il decreto-legge ordinario ${ }^{38}$, che

35 Così Cheli, "L'ampliamento dei poteri normativi dell'esecutivo neiprincipali ordinamenti occidentalin, in Riv. Trim. Dir: Pubbl., 1959, p. 511.

36 Cfr. già Pizzorusso, "I controlli sul decreto-legge in rapporto al problema della forma di governo", in Pol. del Diritto, 1981, p. 302 y s.

37 Cfr. già Galeotti, "Parlamento, Presidente della Repubblica e Governo nel disegno originario e nella realtà attuale della Costituzione", in AA.VV., La Costituzione italiana. Il disegno originario e la realtà attuale, Milano, 1980, p. 177.

38 Modugno, Analisi delle disposizioni della legge 400/88 relative alla potestà legislativa del governo. cit., p. 69 y ss. 
rappresenta «il punto di emersione più difettoso di una democrazia mediatoria e assembleare ${ }^{39}$ : si tratta di un istituto del tutto diverso da quello disciplinato in Costituzione, che costituisce una «bozza di testo ${ }^{40}$, su cui governo, maggioranza e opposizione lavoreranno progressivamente - mediante una reiterazione insistita e spesso contrattata e l'abbondante utilizzo degli emendamenti - dando vita, alla fine, a vere e proprie "forme di colegislazioni governativo-parlamentari" ${ }^{41}$, che il Parlamento è in pratica costretto a convertire (o, quanto meno, a sanare), anche se spesso per ottenere questa approvazione parlamentare il governo non può far altro che porre la questione di fiducia sull'articolo unico del disegno di legge di conversione ${ }^{42}$.

L'effetto sul sistema delle fonti è devastante e, pertanto, la forma di governo ne esce profondamente modificata, superando in maniera nettissima l'assetto delineato nella costituzione formale: si è definitivamente perduta, non senza colpe dello stesso Parlamento, la centralità parlamentare - che anzi viene sostituita da una sorta di "centralità" del decreto-legge - con squilibri e scompensi nella separazione dei poteri e forti impatti su altri valori costituzionalmente tutelati ${ }^{43}$.

L'abuso del decreto-legge indica anche, da altro verso, una profonda crisi del sistema delle fonti ed in particolare un aspetto della crisi della legge, quale fonte del diritto per antonomasia dello Stato moderno ${ }^{44}$. Negli anni, la legge parlamentare si è dimostrata sempre più inadeguata a svolgere il ruolo di fonte-guida del sistema, essendo in ciò affiancata e aggredita da una serie di altre fonti. In particolare, a fronte delle insufficienze della produzione parlamentare, l'esecutivo ha cercato spesso con rimedi peggiori del male - di sostenere la produzione nor-

39 Cosi Di Giovine, "La decretazione d'urgenza in Italia tra paradossi, ossimorie prospettive di riforma", in Studi Parl. Pol. Cost., 1996, p. 9.

40 Sul punto già Paladin, "Gli atti con forza di legge nelle presenti esperienze costituzionali», in Giur. Cost., 1974, p.1525.

41 Così, da ultimo, Pitruzzella, "La lunga transizione: la forma di governo nellXI e nella XII legislatura", in Dir. Pubbl., 1996, p. 429.

$42 \mathrm{Cfr}$. Olivetti, La questione di fiducia nel sistema parlamentare italiano, Milano, 1996 , p. 253 y ss., p. 318 y s.

$43 \mathrm{Da}$ ultimo, Silvestri, Alcuni profili problematici dell'attuale dibattito sui decreti-legge, cit., p. 426 yss.

44 Sul fenomeno, per tutti, Modugno, - Nocilla, Crisi della legge e sistema delle fonti, in Dir. e Società, 1989 , p. 422 y ss. 
mativa usando massicciamente ed impropriamente altre fonti. Non è un caso che alla contrazione della decretazione d'urgenza - a seguito della sentenza n. 360 del 1996 della Corte costituzionale - abbia fatto da contraltare un forte incremento dell'utilizzo della delega legislativa, della delegificazione e di altri poteri regolamentari (soprattutto di c.d. Autorità amministrative indipendenti), nuovi strumenti «abusivi» con cui si tenta di reggere il peso della produzione normativa ${ }^{45}$.

\section{Orientamenti nel controllo della Corte costituzionale sul decreto-legge.}

\subsection{Il sindacato sui presupposti di straordinaria necessità ed urgenza.}

Gli eccessivi abusi del decreto-legge nell'ultimo decennio hanno portato la Corte costituzionale, garante della Costituzione in un sistema accentrato ad accesso soprattutto incidentale (Art. $134^{\circ}$ Cost.), ad interessanti prese di posizione al fine di incrementare le forme del proprio controllo.

Tradizionalmente il giudice costituzionale è sempre apparso assai "timido" nel controllo sui decreti-legge, probabilmente per la ritrosia a controllare valutazioni proprie del circuito politico, come viene tradizionalmente ritenuta quella sulla sussistenza dei presupposti (necessità e urgenza) del decreto-legge.

Negli ultimi anni invece la Corte ha modificato, anche profondamente, i propri indirizzi tradizionali al fine di cercare di contrastare e limitare l'invasività della decretazione governativa d'urgenza.

Per anni la Corte costituzionale, supportata dagli orientamenti della dottrina, ha mostrato "remore consistenti ${ }^{46}$ nell'effettuare un sindacato in ordine all'effettiva sussistenza dei "casi straordinari di necessità ed urgenza».

Dopo essersi in diverse occasioni «rifiutata» di pronunciarsi sul problema, dichiarando inammissibili le relative questioni per contingenti

45 Cfr. Simoncini, Il sistema delle fonti tra governo e parlamento dopo la sentenza n. 360196 della Corte costituzionale, cit, p. 989 y ss.; e Colavitti, "Decretazione d'urgenza e forma di governo", in Dir. e Società, 1999 , p. 341 y ss.

46 Pitruzzella, La legge di conversione del decreto-legge, Padova, 1989, p. 71. 
profili pregiudiziali o processualii ${ }^{47}$ - alla metà degli anni ' 80 - la Corte ha collocato una sorta di "pietra tombale» sul punto, affermando seccamente e ripetutamente che "l'avvenuta conversione in legge del decreto fa ritenere superate le proposte censure», in maniera da ritenere assorbito ogni vizio proprio del decreto nella efficacia novativa della legge di conversione ${ }^{48}$.

Questo orientamento è stato tuttavia modificato a metà degli anni ' 90 , sotto la spinta della prassi sempre più degenerata e delle sollecitazioni della dottrina.

Dapprima, nella sent. n. 29 del 1995, la Corte ha ammesso - come abiter dictum - la possibilità di scrutinare il vizio dei presupposti del decreto-legge, quanto meno nei casi di "evidente mancanza", anche dopo la conversione, negando l'efficacia "sanante» di quest'ultima e sforzandosi invece di ricostruire il difetto della straordinaria necessità ed urgenza quale vizio formale, come tale trasmissibile alla legge parlamentare.

Tale impostazione - pur non avendo mai portato ad annullare un decreto-legge - è stata successivamente ribadita sia rispetto a decreti-legge ancora in corso di conversione (9) $^{4}$ sia rispetto a decreti-legge convertiti in legge ${ }^{50}$, mentre è stata coerentemente esclusa rispetto a disposizioni aggiunte in sede di conversione $e^{51}$ e rispetto a disposizioni di "sanatoria", che si limitano a far salvi gli effetti di decreti non convertiti ${ }^{52}$.

In quest'ottica, è stata prontamente stigmatizzata la sent. n. 360 del 1996, che - precisando come il vizio da reiterazione "può ritenersi sanato quando le Camere, attraverso la legge di conversione (o di sanatoria), abbiano assunto come propri i contenuti o gli effetti della disciplina adottata dal Governo in sede di decretazione d'urgenza»" ${ }^{53}$ sembrava aver negato in via generale la trasmissibilità dei vizi propri del decreto alla legge di conversione ${ }^{54}$.

47 Cfr. sent. n. 55 del 1977, n. 185 del 1981 , e n 34 del 1985 .

48 Cfr. sentt. n. 108 del 1986, n. 243 del 1987, nn. 1033 e 1060 del 1988, n. 263 del 1994; v. anche ordd. nn. 808, 810 e 1035 del 1988.

49 Cfr. sent. n. 161 del 1995 e n. 270 del 1996.

50 Cfr. sent. n. 330 del 1996.

51 Cfr. sent. n. 391 del 1995.

52 Cfr. sent. n. 84 del 1996.

$53 \$ 6$ Considerato in diritto.

54 Cfr. Cicconetti, La sentenza n. 360/1996 della Corte costituzionale e la fine della reiterazione dei decreti-legge: tanto tuonò che piovve, in Giur. Cost., 1996, p. 3165; Romboli, “La reiterazione 
Tuttavia parte della dottrina - prendendo spunto da alcuni obiter dicta contenuti in decisioni di poco successive ${ }^{55}$ - ha cercato di "conciliare» $\mathrm{i}$ due indirizzi della giurisprudenza costituzionale, comparando la natura del vizio da reiterazione con quello da carenza dei presupposti (il primo è meno "grave» riguardando solo una modalità di esercizio di un potere legittimamente attivato; il secondo molto di più, attagliandosi ad una vera e propria carenza di potere, non potendosi neanche attivare il potere di cui all'Art. $77^{\circ}$ Cost. in assenza dei presupposti), e quindi osservando come soltanto il vizio da reiterazione non si trasferisca alla legge di conversione, ma sia punibile esclusivamente in "flagranza", cioè prima dell'intervento parlamentare ${ }^{56}$.

Anche la Corte costituzionale ha poi apertamente ribadito il differente regime dei due vizi (carenza dei presupposti da un lato e reiterazione dall'altro). Nella sent. n. 398 del 1998, infatti - rispetto alla «catena» di decreti-legge in tema di quote-latte, convertita in legge, con sanatoria del pregresso, dopo alcune reiterazioni - la censura per carenza dei presupposti viene esaminata pur trattandosi di decreti-legge convertiti; quella per vizio da reiterazione viene invece rigettata proprio in quanto i decreti-legge in questione sono stati convertiti. In particolare, con riferimento al vizio dei presupposti, se ne ribadisce la rilevabilità in sede di giudizio di costituzionalità, a prescindere dalla conversione, soltanto nei casi di evidente mancanza, cioè quando «essa appaia chiara e manifesta perché solo in questo caso il sindacato di legittimità della Corte non rischia di sovrapporsi alla valutazione di opportunità politica riservata al Parlamenton ${ }^{57}$.

dei decreti-legge decaduti: una dichiarazione di incosituzionalità con deroga per tutti i decreti-legge in corso (tranne uno)", in Foro It., 1996, p. 3274; Pizzorusso, "Ai margini della questione della reiterazione dei decreti-legge: osservazioni su alcuni problemi processualin, in Giur. Cost., 1996, p. 3201; Guastini, "Teoria e dogmatica delle fonti», in Trattato di diritto civile e commerciale diretto da A. Cicu e F. Messineo e continuato da L. Mengoni, Milano, 1998, p. 564.

55 Cfr. ordd. n. 432 del 1996 e n. 90 del 1997 e n. 194 del 1998.

$56 \mathrm{Cfr}$. Romboli, L'efficacia sanante dei vizi formali del decreto-legge da parte della legge di conversione : è davvero cancellata la sent. n. 29 del 1995?, in Giur. Cost., 1997, p. 910 y ss.; Angiolini, «La Corte riapre un occhio sui vizi del decreto-legge convertito?», in Giur. Cost., 1997, p. 2010 y ss.; e volendo Celotto, «Spunti ricostruttivi sulla morfologia del vizio da reiterazione di decreti-leggen, in Gitur. Cost., 1998, p. 1562 ys.

$57 \$ 3$ Considerato in diritto. Cfr. ricostruttivamente Concaro, Il sindacato di costituzionalità sul decreto-legge. Milano, 2000, p. 41 y ss., p. 83 y ss.; e, volendo, Celotto, $L$ '«abusa" del decreto-legge, cit., specie p. 413 yss. 
E' tuttavia da segnalare che, da ultimo, la Corte ha clamorosamente ed inspiegabilmente disatteso questo nuovo orientamento, tornando ad affermare - nella sent. n. 419 del 2000 - che «la conversione in legge [...] ha $[\ldots]$ sanato ogni eventuale vizio attinente al procedimento di formazione del decreto stesso e porta ad escludere l'asserita violazione, nella specie, dell'Art. $77^{\circ}$ Cost. ${ }^{58}$.

A questa decisione, che poteva sembrare anche solo una "svista", hanno fatto seguito ancor più recenti decisioni oscillanti che hanno ora riaffermato (sent. n. 376 del 2001 e sent. n. 16 del 2002) ora negato (sent. n. 29 del 2002) la possibilità di controllare i vizi propri del decreto-legge anche dopo la conversione.

Solo le pronunce che verranno in futuro potranno chiarirci se si tratta di una «svista» isolata oppure di un ulteriore cambio di orientamento della giurisprudenza costituzionale.

\section{2. «Trasferimento" della questione di costituzionalità e «trasferimento» dei vizi del decreto.}

A fronte delle difficoltà pratiche di sindacare un decreto-legge nei sessanta giorni della propria autonoma vigenza, la Corte, a partire dalla sent. n. 84 del 1996 - in linea con gli auspici della dottrina ${ }^{59}$ - ha ammesso la possibilità di trasferire la questione di legittimità costituzionale dalla norma del decreto-legge impugnato alla identica norma del decreto-legge reiterato e attualmente vigente, in maniera da facilitare - stante la massiccia presenza di catene di decreti non convertiti e più volte ripresentati - la possibilità di controllo costituzionale sui decreti-legge. $\mathrm{Si}$ è così superato il vincolo della necessaria instaurazione del giudizio rispetto al singolo decreto della "catena" consentendo, quindi, alla Corte di pronunciarsi anche oltre il breve termine costituzionale di vigenza precaria del singolo atto governativo, a garanzia dei principi di economia processuale e del favor per l'effettività, tempestività e pienezza del controllo costituzionale.

Questo orientamento - fondato sull'affermazione che in linea di principio "la norma contenuta in un atto avente forza di legge vigente al mo-

$58 \$ 9$ Considerato in diritto.

59 Cfr. Tarchi, Incompetenza legislativa del Governo, interposizione del Parlamento e sindacato della Corte costituzionale, cit., p. 967 y s. e Pitruzzella, La legge di conversione del decreto-legge, cit., p. 211 ys. 
mento in cui l'esistenza nell'ordinamento della norma stessa è rilevante ai fini di una utile investitura della Corte, ma non più in vigore nel momento in cui essa rende la sua pronunzia, continua ad essere oggetto dello scrutinio alla Corte stessa demandato quando quella medesima norma permanga tuttora nell'ordinamento con riferimento allo stesso spazio temporale rilevante per il giudizio ${ }^{60}$ - ha trovato fattiva applicazione in relazione allo scrutinio sia di norme contenute in decreti-legge reiterati e ancora vigenti ${ }^{61}$ sia di norme contenute in decreti reiterati e poi convertiti ${ }^{62}$, non solo rispetto a vizi sostanziali della disciplina ma anche rispetto a vizi propri del decreto (carenza dei presupposti, vizio da reiterazione).

In linea di principio, il trasferimento della questione può operare anche rispetto alla clausola di «salvezza» degli effetti dei decreti non convertiti, in quanto la "sanatoria" consiste proprio nel far permanere nell'ordinamento gli effetti prodotti dalla norma poi decaduta, con riferimento allo stesso spazio temporale rilevante per il giudizio.

La giurisprudenza costituzionale ha tuttavia negato il trasferimento rispetto alla legge di "sanatoria", con riferimento alla denuncia dei presupposti costituzionali di necessità ed urgenza del decreto, precisando che «il requisito della necessità ed urgenza va apprezzato con riferimento al singolo decreto-legge, e la censura relativa [...] non è riferibile alla disposizione di sanatoria, che si limita a fare salvi gli effetti del decreto-legge stesso" ${ }^{63}$.

Ad identiche conclusioni la Corte è giunta anche rispetto alla allegabilità del vizio di reiterazione. Tale affermazione è contenuta nella sent. n. 360 del 1996, ove si rileva «che il vizio di costituzionalità derivante dall'iterazione o dalla reiterazione attiene, in senso lato, al procedimento di formazione del decreto-legge in quanto provvedimento provvisorio fondato su presupposti straordinari di necessità ed urgenza: la conseguenza è che tale vizio può ritenersi sanato quando le Camere, attraverso la legge di conversione (o di sanatoria), abbiano assunto come propri i contenuti o gli effetti della disciplina adottata dal Governo in sede di decretazione d'urgenza» ${ }^{64}$.

60 Sent. n. 84 del 1996, \$4.2.3. Considerato in diritto.

61 Sentt. nn. 270 e 360 del 1996.

62 Sentt. nn. 211 del 1997 e 398 del 1998.

63 Così sent. n. 84 del 1996, $\$ 5$ Considerato in diritto; in termini decc. n. 108 del 1996 e n. 263 del 1997.

$64 \$ 6$ Considerato in diritto. 
Sul versante dei vizi è stata quindi esclusa la allegabilità - a «sanatoria» avvenuta - dei vizi propri del decreto, sia per quel che concerne la evidente mancanza dei presupposti, sia per quel che concerne la reiterazione. Senz'altro praticabile deve, invece, ritenersi la scrutinabilità di un vizio materiale del decreto anche una volta che non sia stato convertito ed i suoi effetti siano stati fatti salvi ${ }^{65}$, trasferendo - se del caso - la questione dal decreto-legge impugnato alla clausola di "salvezza" (la Corte, tuttavia, non ha operato automaticamente questo trasferimento, non solo prima della sent. n. 84 del $1996^{66}$, ma anche successivamente, preferendo spesso - ad avviso di chi scrive impropriamente - restituire la questione al giudice a quo per una nuova valutazione della rilevanza a fronte della sopravvenienza della clausola di salvezza ${ }^{67}$ ). Se così non fosse si rischierebbe di creare una "zona franca» da controlli, potendo paradossalmente - il Governo introdurre qualsiasi disciplina, anche palesemente incostituzionale, con un decreto-legge e poi coprirne irrefrangabilmente gli effetti prodotti con clausola di "sanatoria».

\subsection{Il decreto-legge come oggetto di conflitto fra poteri dello Stato.}

Malgrado la dottrina fosse da tempo decisamente orientata nel senso di ammettere l'idoneità degli atti legislativi a dar luogo a conflitto di attribuzione ${ }^{68}$, la Corte costituzionale, con la sent. $n^{\circ} 406$ del 1989 , aveva decisamente negato questa possibilità, insistendo soprattutto sul fatto che la sperimentabilità del conflitto contro tali atti avrebbe costituito un elemento di rottura del nostro sistema di garanzia costituzionale, sistema che, per quanto concerne la legge (e gli atti equiparati), è incentrato sul sindacato incidentale» ${ }^{69}$.

Quest'orientamento negativo è stato tuttavia nettamente ridimensionato, a partire proprio da una fattispecie relativa all'impugnazione di un decreto-legge.

$65 \mathrm{Cfr}$., in fattispecie peraltro particolare, la sent. n. 181 del 1997.

66 Cfr., con perplessità, Nasi, "La Corte costituzionale tra vizi della legge di conversione e vizi della legge di sanatoria ex Art. $77^{\circ}$, ultimo comma, Cost.», in Giur. Cost., 1995, p. 3685 y ss.

67 Cfr. ordd. nn. 128, 230, 317 e 371 del 1997.

68 Da ultimo, ricostruttivamente, Veronesi, I poteri davanti alla Corte. "Cattivo uso" del potere e sindacato costituzionale, Milano, 1999, p. 141 y ss.

$69 \$ 3$ Considerato in diritto. 
Nella sent. n. 161 del 1995 la Corte è arrivata ad un significativo ampliamento delle forme di accesso al giudizio costituzionale, ammettendo l'impugnabilità in conflitto di un decreto-legge proprio al fine di voler tamponare i rischi connessi al dilagare della decretazione d'urgenza. Tale decisione si fonda sul rilievo che l'abuso della decretazione d'urgenza può "assumere connotazioni ancor più gravi nelle ipotesi in cui l'impiego del decreto-legge possa condurre a comprimere diritti fondamentali [e in particolare diritti politici], a incidere sulla materia costituzionale, a determinare - nei confronti dei soggetti privati - situazioni non più reversibili né sanabili anche a seguito della perdita di efficacia della norma. In tali ipotesi, certamente deprecabili il ricorso allo strumento del conflitto tra i poteri dello Stato può dunque rappresentare la forma necessaria per apprestare una difesa in grado di unire all'immediatezza l'efficacia», in quanto il controllo di costituzionalità in via incidentale per il decreto-legge «si presenta di fatto non praticabile in relazione ai tempi ordi-nari del giudizio incidentale ed alla limitata vigenza temporale dello stesso” ${ }^{70}$.

A livello sistematico ricordiamo che successivamente la Corte ha ribadito ed ampliato questo orientamento, ammettendo l'impugnabilità in sede di conflitto anche delle leggi ordinarie e dei decreti legislativi ${ }^{71}$.

70 Sent. $n^{\circ} 161$ del 1995, $\$ 3$ Considerato in diritto; su tale apertura, ampiamente, Carnevale, Decreto-legge all'origine di un conflitto di attribuzioni fra poteri dello Stato? Il sì della Corte costituzionale nella sent. $n .161$ del 1995. Spunti di riflessione, in Modugno, (a cura di), Par condicio e Costituzione, Milano, 1997, p. 143 y ss. e Serges, La sindacabilità degli atti legislativi in sede di conflitto tra poteri nella più recente giurisprudenza costituzionale, ivi, p. 193 ss.

71 Cfr. ord. n. 480 del 1995 e sent. n. 457 del 1999. 